\title{
Using vital signs to assess children with acute infections: a survey of current practice
}

\author{
Matthew Thompson, Richard Mayon-White, Anthony Harnden,
} Rafael Perera, Diane McLeod and David Mant

\author{
ABSTRACT \\ Background \\ GPs are advised to measure vital signs in children \\ presenting with acute infections. Current evidence \\ supports the value of GPs' overall assessment in \\ determining how unwell a child is, but the additional \\ benefit of measuring vital signs is not known. \\ Aim \\ To describe the vital signs and clinical features that \\ GPs use to assess children (aged $<5$ years) with acute \\ infections. \\ Design of study \\ Questionnaire survey. \\ Setting \\ All 210 GP principals working within a 10 mile radius of \\ Oxford, UK.

\section{Method} \\ Data were collected on reported frequency, methods, \\ and utility of measuring vital signs. Description of \\ clinical features was used to assess the overall severity \\ of illness. \\ Results \\ One hundred and sixty-two (77\%) GPs responded. Half \\ $(54 \%, 95 \%$ confidence interval $[\mathrm{Cl}]=47$ to 62$)$ \\ measured temperature at least weekly, compared to \\ pulse $(21 \%, 95 \% \mathrm{Cl}=15$ to 27$)$, and respiratory rates \\ $(17 \%, 95 \% \mathrm{Cl}=11$ to 23$)$. Almost half of GPs $(77$, \\ $48 \%)$ never measured capillary refill time. Temperature \\ was measured most frequently using electronic aural \\ thermometers (131/152; $86 \%)$; auscultation or counting \\ were used for pulse and respiratory rates. A minority \\ used pulse oximeters to assess respiratory status \\ (30/151, 20\%). GPs' thresholds for tachypnoea were \\ similar to published values, but there was no \\ consensus on the threshold of tachycardia. \\ Observations of behaviour and activity were \\ considered more useful than vital signs in assessing \\ severity of illness.

\section{Conclusion} \\ Vital signs are uncommonly measured in children in \\ general practice and are considered less useful than \\ observation in assessing the severity of illness. If \\ measurement of vital signs is to become part of \\ standard practice, the issues of inaccurate \\ measurement and diagnostic value need to be \\ addressed urgently. \\ Keywords \\ body temperature; child; heart rate; infection; \\ observation; triage.
}

\section{INTRODUCTION}

Acute infections are one of the most common reasons why children are seen by GPs. One of the core tasks of primary care is to distinguish children with a possible serious infection from the vast majority who have minor or self-limiting infections. Only $1-3 \%$ of children with acute infections seen by GPs during surgery and out-of-hours are referred to hospital specialists for urgent assessment or admission. ${ }^{1,2}$ This implies that the vast majority of children are managed adequately in primary care. However, children with serious bacterial infections are sometimes misdiagnosed in primary care; up to $50 \%$ of children with meningococcal disease were missed at first consultation with GPs..$^{3-5}$

The clinical methods that GPs use to identify children with serious bacterial infection have been described in qualitative studies..$^{6-8} \mathrm{~A}$ feeling that 'something is wrong' is a useful discriminating factor in children presenting to primary care with acute infection. ${ }^{9}$ In comparison, there is little published evidence about the extent to which vital signs are measured or their value in children with acute illness in routine general practice. In 1992, a survey of thermometer use reported that $48 \%$ of GPs measured

M Thompson, $M B C h B, D P h i l$, clinical lecturer in general practice; $\mathbf{R}$ Mayon-White, FRCP, FFPH, epidemiologist; A Harnden, FRCGP, FRCPCH, university lecturer in general practice; $\boldsymbol{R}$ Perera, DPhil, medical statistician; D McLeod, RGN, research nurse; D Mant, FRCP FRCGP, professor of general practice, Oxford University Department of Primary Health Care, Oxford.

Address for correspondence

Dr Matthew Thompson, Oxford University Department of Primary Health Care, Old Road Campus, Oxford, OX3 7LF. E-mail: matthew.thompson@dphpc.ox.ac.uk

Submitted: 25 July 2007; Editor's response: 1 August 2007; final acceptance: 5 February 2008.

(C) British Journal of General Practice.

This article was originally online first. Cite this article as: Br J Gen Pract 2008; 58: 236-241. Advance online publication. DOI: $10.3399 /$ bjgp08X279689 
temperature less than once per fortnight. ${ }^{10}$ Despite the paucity of evidence, the National Institute for Health and Clinical Excellence (NICE) has recommended that temperature, heart rate, respiratory rate, and capillary refill time are measured in all children presenting to primary care with febrile illness. ${ }^{1}$

This study surveyed how GPs measure and use vital signs in pre-school children presenting with acute infections, and compared their utility with the clinical features used to assess a child's respiratory status and their overall severity of illness.

\section{METHOD}

\section{Design, setting and participants}

The survey was conducted by post between February and April 2006. A list of GP principals in a 10 mile radius of Oxford city centre was obtained from the Thames Valley Primary Care Agency. The list identified 227 GPs in 46 practices, serving a combined practice population of 360800 . Each practice was contacted by phone to verify that the list was correct; 17 GPs were found to be ineligible or unable to participate due to long-term leave $(n=6)$, maternity leave $(n=1)$, not a partner $(n=4)$, left the practice $(n=4)$, or long-term sick leave $(n=2)$. The eligible population surveyed was therefore 210. A reminder letter and questionnaire were sent to those GPs who had failed to respond within 6 weeks; each responder received a small payment of $£ 40$ for the time taken to complete the survey.

\section{Questionnaire content}

The questionnaire elicited the methods and frequency with which practitioners measured vital signs (temperature, heart rate, respiratory rate, and capillary refill), and the value of the vital sign that they would consider abnormal in children aged 5 years and younger. Free-text responses were used to elicit the clinical features they used when assessing a child's respiratory status, and when making a 'global' or overall assessment of their severity of illness. No attempt was made to validate GPs' responses from their patient records. GPs were also asked to rate on a five-point Likert scale ( $1=$ never useful, $5=$ extremely useful) how useful they felt it was to measure temperature, pulse rate, and respiratory rate when managing a child in 10 different scenarios:

a child who looks generally unwell and I am worried they might have a serious infection;

when I refer a child to hospital for assessment/ admission for an acute illness;

to reassure the parents;

on my own children when they have infections;

if I suspect influenza;

a child with breathing difficulties;

\section{How this fits in}

GPs use an overall or global assessment to help them identify children with serious infections, but the extent to which they also use vital signs is not known. This study found that GPs generally measure vital signs infrequently (with the exception of temperature), and perceive them as less useful than observing activity, behaviour, or work of breathing in assessing children of pre-school-age with acute infections. If measurement of vital signs is to become standard clinical practice, more accurate measurement technologies and evidence for their diagnostic value are needed.

a child aged 1 month with possible infection; a child aged 1-3 months with possible infection; in order to assess level of dehydration; and a child with abnormal skin colour.

The results from two of these scenarios were selected to determine whether the perceived usefulness of vital signs differed in children who the authors considered to be at the opposite ends of the spectrum of clinical severity of illness:

a child who looks generally unwell and I am worried they might have a serious infection (that is, a generally unwell child); and

to reassure the parents (that is, a generally well child).

\section{Analysis}

The data were analysed with SPSS (version 14.0). The statistical significance of associations between categorical variables was determined using the $\chi^{2}$ test or Fisher's exact test; 95\% confidence intervals (Cl) were based on the standard error of a proportion. Independent sample $t$-tests or analysis of variance (ANOVA) were used to assess the significance of differences in means of continuous parametric variables, or the Mann-Whitney $U$ test for nonparametric variables. If responders gave a range rather than a single threshold value for the temperature, pulse rate, or respiratory rate they considered abnormal, the midpoint of the range was used. Freetext responses were grouped into categories according to a coding schedule with any ambiguity or

Table 1. Reported frequency of measurement of temperature, pulse, and respiratory rate in children aged $<5$ years in routine clinical practice ( $n=162$ GPs).

\begin{tabular}{lccc} 
Frequency of measurement & $\begin{array}{c}\text { Temperature, } \\
n(\%)\end{array}$ & $\begin{array}{c}\text { Pulse rate, } \\
n(\%)\end{array}$ & $\begin{array}{c}\text { Respiratory rate, } \\
n(\%)\end{array}$ \\
\hline Never & $10(6.2)$ & $11(6.8)$ & $11(6.8)$ \\
\hline Less than once a month & $24(14.8)$ & $63(38.9)$ & $53(32.7)$ \\
\hline Between once a month and once a week & $40(24.7)$ & $54(33.3)$ & $71(43.8)$ \\
\hline At least once a week & $88(54.3)$ & $34(21.0)$ & $27(16.7)$ \\
\hline
\end{tabular}




Table 2. Clinical features used by GPs in assessing overall
severity of infection in children ( $\boldsymbol{n}=162$ GPs).
\begin{tabular}{llc} 
Rank & Clinical feature & $\begin{array}{c}\text { Number of responses } \\
\text { a }\end{array}$ \\
\hline 1 & Activity or behaviour & $148(91.3)$ \\
\hline 2 & Skin colour (pallid, mottled, blotchy, cyanosed) & $108(66.7)$ \\
\hline 3 & Temperature & $103(63.6)$ \\
\hline 4 & Respiratory rate & $85(52.5)$ \\
\hline 5 & Seeking specific focus of infection & $83(52.1)$ \\
\hline 6 & Level of hydration & $62(38.3)$ \\
\hline 7 & Peripheral circulation (capillary refill, & $62(38.3)$ \\
\hline 8 & peripheral perfusion, cold extremities) & $60(37.0)$ \\
\hline 9 & Pulse rate & $44(27.2)$ \\
\hline 10 & Body tone or handling & $18(11.1)$ \\
\hline aParental concern was ranked 11th (15, 9.3\%) and non-specific intuition 12 th (11,6.8\%).
\end{tabular}

other difficulty in categorising a free-text response resolved by discussion between the authors.

\section{RESULTS}

\section{Responders}

Responses were received from 162 GPs (77\%). Five responders declined to give identifying information, but the remaining 157 were similar to the 48 nonresponders in terms of mean number of years since graduation (22.5 versus 22.8), female sex (51.2\% versus $50.0 \%$ ), size of practice (9354 versus 9807 patients), and working in a teaching practice $(83.3 \%$ versus $83.3 \%$ ).

\section{Frequency of measuring vital signs}

Table 1 shows how frequently GPs reported measuring temperature, pulse rate, and respiratory rate in the previous 12 months. Temperature was the vital sign measured most frequently; more than half

\section{Table 3. Clinical features used by GPs in assessing severity} of respiratory illness in children $(n=162)$.

\begin{tabular}{llc} 
Rank & Clinical feature & $\begin{array}{c}\text { Number of } \\
\text { responses }\end{array}$ \\
\hline 1 & Work of breathing & $n(\%)$ \\
\hline 2 & Respiratory rate & $140(86.4)$ \\
\hline 3 & Skin colour (that is, pallor or cyanosis) & $114(70.4)$ \\
\hline 4 & Activity or behaviour & $64(39.5)$ \\
\hline 5 & Auscultation of chest & $42(25.9)$ \\
\hline 7 & Non-specific 'respiratory distress' & $30(18.5)$ \\
\hline 8 & Pulse rate & $21(13.0)$ \\
\hline 9 & Oxygen saturation & $15(9.3)$ \\
\hline 10 & Presence of stridor & $13(8.0)$ \\
\hline & Temperature & $11(6.8)$ \\
\hline
\end{tabular}

(54\%, $95 \% \mathrm{Cl}=47$ to 62$)$ reported measuring it at least once per week, compared to $21 \%(95 \% \mathrm{Cl}=15$ to 27$)$ for pulse, and $17 \%(95 \% \mathrm{Cl}=11$ to 23$)$ for respiratory rate. Less than $7 \%$ of practitioners reported never measuring pulse, temperature, or respiratory rate. In contrast, less than half of responders $(77,48 \%)$ reported that they never measured capillary refill time.

\section{Methods of measuring vital signs}

The methods used by GPs to determine whether a child had fever included measuring with a thermometer (139/162, 86\%), touching the child $(78 / 162,48 \%)$, or relying on the parents' observation of fever (44/162, $27 \%)$. Among the $152 \mathrm{GPs}$ who measured temperature using a thermometer, the device used most frequently was an electronic aural thermometer (131/152, 85\%); most of the other practitioners used other electronic devices (oral $n=5$, axillary $n=6$ ), although six practitioners used forehead colour-changing strips. GPs who used thermometry alone to assess temperature tended to be qualified more recently than those who used other methods (mean time since qualification 20.0 years versus 24.8 years, $P<0.001$ ). Among the 151 GPs who measured heart rate, the most common method was auscultation of the heart (143/151, 95\%). Similarly, among the 151 GPs who measured respiratory rate, the usual method was by counting breaths using observation (149/151, 99\%). A minority of GPs reported that they used a pulse oximeter to measure pulse rate $(13 / 151,9 \%)$, or to assess respiratory status (30/151, 20\%).

\section{Thresholds for abnormality of vital signs}

Almost all $(152,94 \%)$ responders cited a temperature threshold that they considered to be abnormal (that is, fever); $33(22 \%)$ cited $37.0^{\circ} \mathrm{C}, 65(43 \%) 37.1-37.5^{\circ} \mathrm{C}$, $47(31 \%) 37.6-38.0^{\circ} \mathrm{C}$, and seven $(5 \%)$ a threshold of $>38.0^{\circ} \mathrm{C}$. Half $(86,53 \%)$ of GPs provided a threshold for tachycardia. The median thresholds for tachycardia were $130 \mathrm{beats} / \mathrm{min}$ (bpm; range $80-200 \mathrm{bpm}$ ) for infants $<1$ year, $110 \mathrm{bpm}$ (range $80-160 \mathrm{bpm}$ ) for children 1-2 years, and $100 \mathrm{bpm}$ (range $70-150 \mathrm{bpm}$ ) for children 2-5 years. A greater proportion of practitioners $(140,86 \%)$ quoted a threshold for tachypnoea. The median thresholds cited for tachypnoea were $40 \mathrm{bpm}$ (range 20-100 bpm) for infants $<1$ year, $30 \mathrm{bpm}$ (range 20-90 bpm) for those $1-2$ years, and $30 \mathrm{bpm}$ (range 15-80 bpm) for children $2-5$ years of age.

\section{Clinical features used in global assessment}

Table 2 summarises the clinical features that GPs reported as being most useful for assessing overall severity of illness. Observing the child's level of activity or behaviour was the clinical feature cited most 
frequently by GPs $(148,91 \%)$. The specific features of activity or behaviour that GPs described in the freetext comments included their level of alertness (104, $70 \%$ ), response to social interaction including eye contact or engaging with the doctor $(58,39 \%)$, and/or their interest in the surroundings such as playing or moving around the consultation room (29, 20\%). Temperature and respiratory rate were ranked third and fourth in importance, after skin colour. Pulse rate was considered the least useful of the vital signs, less important than assessing peripheral circulation.

\section{Clinical features used in assessing respiratory illness}

Table 3 shows the clinical features that GPs cited as being most useful in assessing the severity of respiratory illness. Work of breathing was the feature reported most frequently $(140,86 \%)$, followed by respiratory rate. The specific components of work of breathing described in the free-text responses were intercostal or subcostal recession (111/140, 79\%), followed by non-specific accessory muscle use (52, $37 \%)$, tracheal tug $(23,16 \%)$, grunting (14, 10\%), and/or nasal flare $(9,6 \%)$. Among the 42 GPs who cited activity or behaviour as being useful, the most common features cited in the free-text comments were difficulty feeding or talking $(23,16 \%)$, followed by tiredness or exhaustion (18, 13\%).

\section{Usefulness of vital signs in different clinical situations}

GPs' rating of how useful they felt measuring temperature, heart rate, and respiratory rate were in clinical management are displayed in Figure 1. For a generally unwell child, a usefulness score of $\geq 4$ was given by $74 \%(95 \% \mathrm{Cl}=67$ to 81$)$ of GPs for measuring temperature, $68 \%(95 \% \mathrm{Cl}=61$ to 75$)$ of GPs for heart rate, and $70 \%(95 \% \mathrm{Cl}=63$ to 77$)$ of GPs for respiratory rate. In contrast, for a generally well child, a usefulness score of $\geq 4$ was given by $57 \%$ ( $95 \% \mathrm{Cl}=50$ to 65$)$ of GPs for measuring temperature, $24 \%(95 \% \mathrm{Cl}=18$ to 31$)$ of GPs for pulse rate, and $28 \%(95 \% \mathrm{Cl}=21$ to 35$)$ of GPs for respiratory rate.

The usefulness ratings were compared between GPs who measured vital signs frequently (that is, at least once per week), and those who measured them infrequently (that is, less than once per year or never). The 88 GPs who measured temperature frequently rated it as significantly more useful than the 34 who measured it infrequently for children who were generally unwell (mean scores 4.4 versus 2.5, $P<0.001)$, as well as children who were generally well (3.9 versus $2.4, P<0.001$ ). The $34 \mathrm{GPs}$ who measured pulse rate frequently rated it as significantly more useful than the 73 who measured it infrequently for a generally unwell child (4.1 versus $3.5, P=0.005)$ only.
Figure 1. Perceived usefulness of measuring vital signs in children aged $<5$ years. Responders were asked to rate usefulness of measuring vital signs in 'a child who looks generally unwell and I am worried they might have a serious infection' (generally unwell child), and 'to reassure the parents' (generally well child).

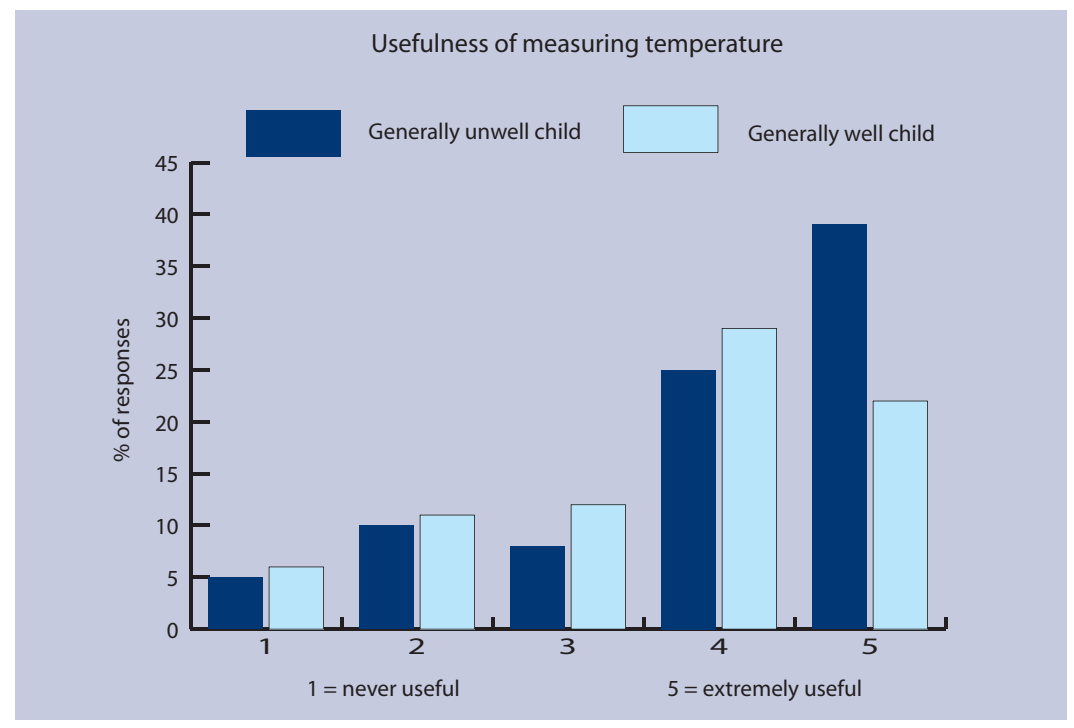

Usefulness of measuring pulse rate

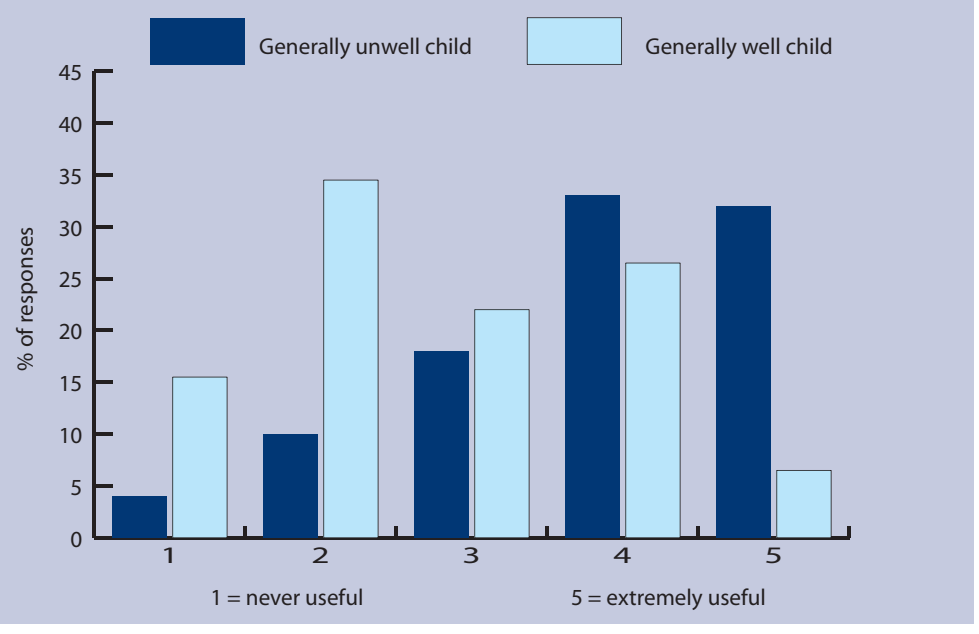

Usefulness of measuring respiratory rate

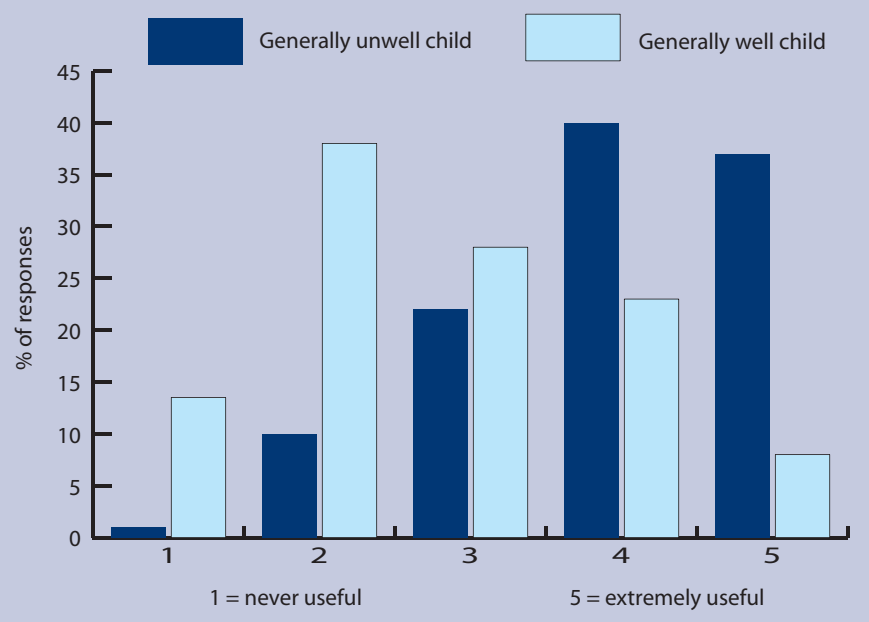


Finally, the 27 GPs who measured respiratory rate frequently rated it as significantly more useful than the 62 GPs who measured it infrequently for children who were generally unwell (4.4 versus $3.6, P=0.001$ ) as well as children who were generally well (3.0 versus $2.4, P=0.007)$.

\section{DISCUSSION}

\section{Summary of main findings}

With the exception of temperature, vital signs were uncommonly measured by the GPs in this survey. Modern technology was commonly used for temperature measurement, but pulse oximetry was uncommon in the practices studied, where GPs relied on the traditional methods of counting heart beats and inspiratory movements. Responders displayed wide variation in their perception of what are considered abnormal thresholds of temperature, heart rate, and respiratory rate. GPs who reported that they found vital signs more useful were also more likely to report measuring them more frequently. However, they ranked activity or behavioural features, abnormal skin colour, and increased work of breathing as more useful than vital signs in assessing children with acute infections.

\section{Strengths and limitations of the study}

The high response rate, and similarity in known characteristics of responders and non-responders, suggests that these results are generalisable to all GPs in the survey area. The survey area is recognised to provide a high standard of general practice care, with high levels of involvement in educational activities and provision of out-of-hours care. Therefore, it is likely that the results are broadly characteristic of best current practice in the UK. It was not possible to validate GPs' responses from their patient records. Differences between reported and actual clinical practices could lead to an over- or under-estimation of frequency or perceived usefulness of vital signs. The survey was carried out in the springtime which may have increased GPs' recollections of the frequency with which they had measured vital signs in children in the previous 12 months.

\section{Comparison with existing literature}

The key question is whether the current practice of seldom measuring vital signs in children, and giving more weight to activity and behavioural features is in fact best practice. A recent NICE guideline reviewed the literature on the assessment of young children with feverish illness, and recommended that vital signs should be a routine part of assessing febrile children. ${ }^{11}$ This guideline identified strong evidence from prospective cohort studies that rapid respiratory rate was associated with serious illness in children (including pneumonia), and that height (but not duration) of fever was associated with increased risk of serious illness especially in infants under 6 months of age. It also found that the overall sensitivity of prolonged capillary refill time for serious illness or dehydration was only modest (60\%), although in children with a higher risk of serious disease (that is, those with petechial rash), a refill time $\geq 3$ seconds was strongly associated with meningococcal disease (odds ratio 29). In contrast it found 'a lack of evidence regarding heart rate as a marker of serious illness', but recommended routine heart rate measurement based on Delphi consensus.

The present study suggests that vital signs are not measured routinely, but they are perceived as being more useful in children who appear to be generally unwell. There is evidence to support the view that global assessment of behaviour and responsiveness does have important diagnostic value in assessing sick children. McCarthy, et al showed 25 years ago that overall assessment of young febrile children, particularly of impaired responsiveness (for example, not looking at the observer; not looking round the room), was a powerful predictor of serious illness. ${ }^{12}$ Subsequent qualitative studies of how GPs recognise serious bacterial infections have described the observational items that they use, such as level of activity, facial expression, eye contact, pallor, and crying characteristics. ${ }^{6-8}$ Furthermore, a recent prospective study in Belgium found that GPs' feeling of 'something is wrong' was the strongest predictor of serious infection among children presenting in primary care with acute infections. ${ }^{9}$ The present findings add weight to these studies by describing in detail the relative frequency and perceived usefulness of these features. Knowledge of these observational features should now be a standard component of the training of primary care practitioners.

The main arguments against measuring vital signs are the limited time available in a brief consultation, the difficulty of achieving accurate readings, and lack of evidence for their predictive value in primary care. To detect fever, touch has a sensitivity of up to $94 \%$, but the aural thermometers favoured by the clinicians surveyed are less sensitive in clinical use, ${ }^{13,14}$ probably because of user errors and variations in the external auditory canal..$^{15}$ The height of fever has relatively poor sensitivity for identifying serious infection, which may explain responders' wide variation in definitions of fever. In contrast, tachypnoea is a useful sign of pneumonia or dehydration in infants. ${ }^{16,17}$ However, measurement of respiratory rate is associated with high inter-observer variability, ${ }^{16,18}$ probably because accurate measurement requires one minute of observation - a relatively long time in a primary care consultation. ${ }^{19}$ Nevertheless, GPs' thresholds for tachypnoea were 
very close to published definitions of abnormal respiratory rate (for example, Advanced Paediatric Life Support guidelines). Tachycardia is a cardinal feature of fever itself, as well as the systemic inflammatory response syndrome. However, the current 'cut-off' levels of heart rate are based on scant evidence, and do not take into account the effect of temperature on heart rate. ${ }^{20}$ This may explain why GPs considered heart rate to have limited importance, and cited such wide variations in the thresholds for tachycardia.

\section{Implications for future research and clinical practice}

It should be possible to make better use of the new measurement technologies to increase accuracy of vital signs, although their application is not straightforward. For example, very low oxygen saturation assessed by pulse oximetry is a good predictor of the need for urgent admission (for example, odds ratio 12 for arterial oxygen saturation $\left[\mathrm{SaO}^{2}\right]$ of $\left.88 \%\right){ }^{21}$ However, most children seen in general practice will still be well enough to compensate (by rapid breathing) for their respiratory pathology, and the values of $95-100 \%$ most often found in general practice have little diagnostic value. ${ }^{21,22}$ In order to give clinicians better guidance on interpreting values of vital signs, further research is needed to determine the threshold levels that will provide the optimum operating characteristics of each vital sign (or combination of vital signs). If the current problem of inaccurate measurement can be also be overcome, there are three arguments in favour of measuring vital signs in general practice in addition to making a global overall assessment. The first is the empirical observation cited in the introduction that children with serious infection are missed in general practice. If the diagnostic (or prognostic) value of vital signs for identifying early changes in cardiorespiratory status can be clearly demonstrated, they may improve the sensitivity of identifying serious infections. Secondly, an increasing proportion of out-of-hours care is provided by healthcare workers with less paediatric training than GPs, and who may not have the experience to make good assessments based on overall appearance. Thirdly, a key aspect of diagnosis in general practice is assessing change over time. It is much easier to assess clinical change if objective measurements have been accurately made and recorded.

In conclusion, current practice is to measure vital signs infrequently, with the exception of temperature. The methods used for measurement and the definitions of normal values are of questionable accuracy. If measurement of vital signs is to become an accepted part of the good clinical care of children, then accurate measurement techniques and evidence for their diagnostic value in primary care are needed.

\section{Funding body}

This survey was funded by NHS Research and Development funding from the Thames Valley Primary Care Research Partnership

\section{Competing interests}

The authors have stated that there are none

\section{Acknowledgements}

We gratefully acknowledge the assistance of Sue Smith (research manager), Sue Rous and Gerry Jewell (research nurses), and particularly the GPs who took time to respond to the survey.

\section{Discuss this article}

Contribute and read comments about this article on the Discussion Forum: http://www.rcgp.org.uk/bjgp-discuss

\section{REFERENCES}

1. Avery AJ, Groom L, Boot D, et al. What problems do patients present with outside normal general practice surgery hours? A prospective study of the use of general practice and accident and emergency services. J Public Health Med 1999; 21(1): 88-94.

2. MacFaul R. The health of children and young people. London: Office for National Statistics, 2004.

3. Thompson MJ, Ninis N, Perera R, et al. Clinical recognition of meningococcal disease in children and adolescents. Lancet 2006367 : 397-403.

4. Sorensen HT, Moller-Petersen J, Krarup HB, et al. Diagnostic problems with meningococcal disease in general practice. J Clin Epidemiol 1992; 45(11): 1289-1293.

5. Nadel S, Britto J, Booy R, et al. Avoidable deficiencies in the delivery of health care to children with meningococcal disease. J Accid Emerg Med 1998; 15(5): 298-303.

6. Granier S, Owen P, Pill R, Jacobson L. Recognising meningococcal disease in primary care: qualitative study of how general practitioners process clinical and contextual information. BMJ 1998; 316(7127): 276-279.

7. Brennan CA, Somerset M, Granier SK, et al. Management of diagnostic uncertainty in children with possible meningitis: a qualitative study. $\mathrm{Br}$ Gen Pract 2003; 53(493): 626-631.

8. Van den Bruel A, Bruyninckx R, Vermeire E, et al. Signs and symptoms in children with a serious infection: a qualitative study. BMC Fam Pract 2005; 6: 36.

9. Van den Bruel A, Aertgeerts B, Bruyninckx R, et al. Signs and symptoms for diagnosis of serious infections in children: a prospective study in primary care. Br J Gen Pract 2007; 57(540): 538-546.

10. Clarke S. Use of thermometers in general practice. BMJ 1992; 304(6832): 961-963.

11. National Institute for Health and Clinical Excellence. Feverish illness in children - assessment and initial management in children younger than 5 years. London: National Institute for Health and Clinical Excellence, 2007.

12. McCarthy PL, Jekel JF, Stashwick CA, et al. Further definition of history and observation variables in assessing febrile children. Pediatrics 1981; 67(5): 687-693.

13. Whybrew K, Murray M, Morley C. Diagnosing fever by touch: observational study. BMJ 1998; 317(7154): 321.

14. Hay AD, Peters TJ, Wilson A, Fahey T. The use of infrared thermometry for the detection of fever. Br J Gen Pract 2004; 54(503): 448-450

15. Crawford DC, Hicks B, Thompson MJ. Which thermometer? Factors influencing best choice for intermittent clinical temperature assessment. $J$ Med Eng Technol 2006; 30(4): 199-211.

16. Margolis P, Gadomski A. The rational clinical examination. Does this infant have pneumonia? JAMA 1998; 279(4): 308-313.

17. Lynch T, Platt R, Gouin S, et al. Can we predict which children with clinically suspected pneumonia will have the presence of focal infiltrates on chest radiographs? Pediatrics 2004; 113(3 part 1): e186-e189.

18. Hay AD, Wilson A, Fahey T, Peters TJ. The inter-observer agreement of examining pre-school children with acute cough: a nested study. BMC Fam Pract 2004; 5: 4

19. Simoes EA, Roark R, Berman S, et al. Respiratory rate: measurement of variability over time and accuracy at different counting periods. Arch Dis Child 1991; 66(10): 1199-1203.

20. Hanna CM, Greenes DS. How much tachycardia in infants can be attributed to fever? Ann Emerg Med 2004; 43(6): 699-705.

21. Keahey L, Bulloch B, Becker AB, et al. Initial oxygen saturation as a predictor of admission in children presenting to the emergency department with acute asthma. Ann Emerg Med 2002; 40(3): 300-307.

22. Tanen DA, Trocinski DR. The use of pulse oximetry to exclude pneumonia in children. Am J Emerg Med 2002; 20(6): 521-523. 